\title{
The idle resource items workload and implication on different weight balance rate in grid scheduling
}

\begin{abstract}
This paper discusses the impact of background workload for idle re-source items of computing elements in grid computing. Most of the previous re-search did not consider this factor. A resource item may be processing local system operations when the grid perceives them to be idle, thus upsetting grid processing activities. The introduction of the resource items and background load factor in this study will reveal the true computing capability of computing elements. This background load factor, represented in the form of weightage on resource item, is tested to seek overall grid performance. By allocating the right balance of workload weightage of resource item in a computing element, a significant improvement in processing performance is achieved.
\end{abstract}

Keyword: Scheduling; Resource item; Background load; Grid computing; Idle; Workload 